\title{
EXPERIMENTAL INVESTIGATION OF STATISTICAL MOMENTS OF TRAVEL TIME IN GRID-GENERATED TURBULENCE
}

\author{
T.A. Andreeva ${ }^{*}$, S.B. Meleshi ${ }^{\dagger}$ and W.W. Durgin $\neq$ \\ Mechanical Engineering Department, Worcester Polytechnic Institute, Worcester, MA, 01609
}

\begin{abstract}
An experimental technique for investigation of the behavior of acoustic wave propagation through a turbulent medium is discussed. The present study utilizes the ultrasonic travel-time technique to diagnose a grid-generated turbulence. The statistics of the travel-time variations of ultrasonic wave propagation along a path are used to determine some metrics of the turbulence. Experimental data obtained using ultrasonic technique confirms numerical and theoretical predictions of nonlinear increase of the first-order travel time variance with propagation distance.
\end{abstract}

\section{INTRODUCTION}

The classical theory of acoustic wave propagation through turbulence predicts linear increase of the firstorder travel time variance with the propagation distance $^{1}$. However, recent numerical and theoretical studies exhibit an almost quadratic growth of travel time variance with travel distance $e^{2,3,4}$. The reason for this behavior is not entirely understood yet, but it seems to be closely related to the occurrence of first caustics ${ }^{5,6,7,8}$. If a wave propagates in a random medium, then at some distance $x$ from the source, caustics appear. The information about the distance is important. For example, some tomographic methods used for remote sensing of the atmosphere and the ocean are based on the ray acoustics approach so that knowledge of the distance to the caustics will define the region of applicability of the tomography methods. ${ }^{9,10,11}$ The higher the turbulence intensity, the shorter the distance at which the first caustic occurs. Codona et $\mathrm{al}^{6}$ demonstrated the importance of the occurrence of caustics when evaluating the average travel time in a random scalar field. The probability of the appearance of the caustics in a random field was theoretically explored by Kulkarny and White, ${ }^{6}$ Klyatskin, ${ }^{12}$ Blanc-

\footnotetext{
* Postdoctoral Fellow, tatiana@wpi.edu

${ }^{\dagger}$ Graduate student, airshang@woi.edu

‡ Professor, Associate Fellow, wwdurgin@wpi.edu
}

Benon et al. ${ }^{7}$ Numerically the phenomena of caustics was studied by Blanc-Benon and Juvé, ${ }^{8}$ Juvé et $\mathrm{al}^{2}{ }^{2}$ Karweit et al. ${ }^{3}$

The ultrasonic technique for measuring flows offers great potentials for turbulent flow diagnostics. ${ }^{14}$ The techniques employed have been used in our previous work. ${ }^{15}$ Wherein we considered a locally isotropic velocity field, which is realized by introducing a grid in a uniform flow. ${ }^{16}$

In the present study two features are noteworthy. First, the experimental investigation is performed under well-controlled laboratory conditions. Second, the data acquisition and control system featured a very highspeed analog to digital conversion card that enabled excellent resolution of ultrasonic signals. The work aims to investigate the influence of the grid-generated turbulent flow on acoustic wave propagation in terms of the variance of the travel time. Comparison with numerical predictions by Juvé et al, 1991; Karweit et al, 1991, as well as theoretical estimations by Chernov, 1961 and Iooss et al, 2000, complement the study.

\section{EXPERIMENTAL ARRANGEMENTS AND}

\section{RESULTS}

In the experimental part of the study we utilize ultrasonic pulses traveling in straight paths as shown in Figure 2.1. The sound propagates across a gridgenerated turbulence from a transmitter to a receiver separated by a distance $L$ in the direction perpendicular to the mean flow. The sound propagates across a gridgenerated turbulence from a transmitter to a receiver 
separated by a distance $L$ in the direction perpendicular to the mean flow.

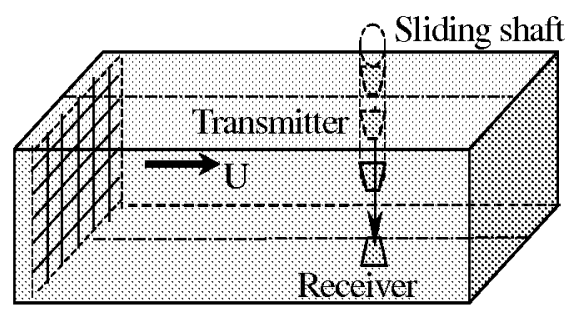

Figure 2.1 Sketch of the wind-tunnel test section with ultrasonic flowmeter.

The mean velocity is fixed at $18 \mathrm{~m} / \mathrm{s}$, which corresponds to the Reynolds number, based on the mesh size, $\operatorname{Re} \approx 7200$. The travel path $L$ is changed by displacing the transducer-transmitter along path wave. To insure the aliment and accurate distance measurement the transmitter-transmitter is placed in the sliding shaft, as shown in Figure 1.

The flowmeter equation may be used to derive an expression for a travel time of a wave traveling from the speaker to microphone $t$.

$$
t=\int_{0}^{L} \frac{d y}{c-u} \approx t_{0}+\frac{1}{c^{2}} \int_{0}^{L} u d y, u=U \cos \vartheta+u^{\prime}
$$

where $t_{0}$ is a travel time in the undisturbed media, $U$ is a mean velocity, $c$ is a sound speed, $u^{\prime}$ are fluctuations of the mean flow velocity, and $\vartheta$ is an angle between the sound path and a mean velocity vector. In the considered case $\vartheta=90^{\circ}$. In Equation (1) we neglected the terms of order $u^{2} / c^{2}$ and higher. In the experiment the only parameter that is measured is a travel time of ultrasound pulses $t(L)$. The data from experiments performed for a finite number of different lengths $L, L^{\prime}, L^{\prime \prime} \ldots$ are collected. For each of those lengths a variance of a travel time is calculated as

$$
\sigma_{t}=\left\langle(t-\langle t\rangle)^{2}\right\rangle
$$

where triangular brackets indicate time averaging.

The experiments were carried out in the $11.75^{\prime \prime} \times 11.62^{\prime \prime} \times 45.25^{\prime \prime}$ test section of low turbulence, low speed open circuit type wind tunnel. Experimental data were obtained for ultrasonic wave propagation downstream of a grid in the wind tunnel. Such gridgenerated turbulence is well characterized and features a mean flow with superimposed velocity fluctuations.
The ultrasonic path was perpendicular to the mean flow direction as shown in Figure 1. Path lengths were changed from $0.05 \mathrm{~m}$ to $0.18 \mathrm{~m}$ and the transducers were of $100 \mathrm{kHz}$ working frequency. The grid size, M, was $6 \cdot 10^{-3} \mathrm{~m}$. The measurements were collected at $0.63 \mathrm{~m}$ downstream the grid. The mean flow velocity $U$ was $18 \mathrm{~m} / \mathrm{s}$. The Reynolds number $\operatorname{Re}_{M}$ based on $M$ and $U$ was about 7200 . Transmitted and received signals in a digital form were transmitted and collected respectively using high speed data acquisition cards (DAQ). The instant of the transmitted wave departure was registered with high precision by National Instrument Data Acquisition Card (DAQ). The received wave was registered/digitized and stored by the CompuScope $82 \mathrm{G}$ DAQ with large acquisition memory. Digital representation of the experimental data, provided by the DAQs allowed determination of the travel time $t$ very precisely. A more detailed description of the experimental particulars may be found in Andreeva and Durgin. ${ }^{15}$ Figure 2.2 demonstrates data representation obtained from CompuScope 82G DAQ, transferred to the PC and processed in Excel. The acquisition rate was 50000000 samples/s. Two signals shown in Figure 2.2 are transmitted four square-wave burst signal $e_{1}$ and received signal $e_{2}$.

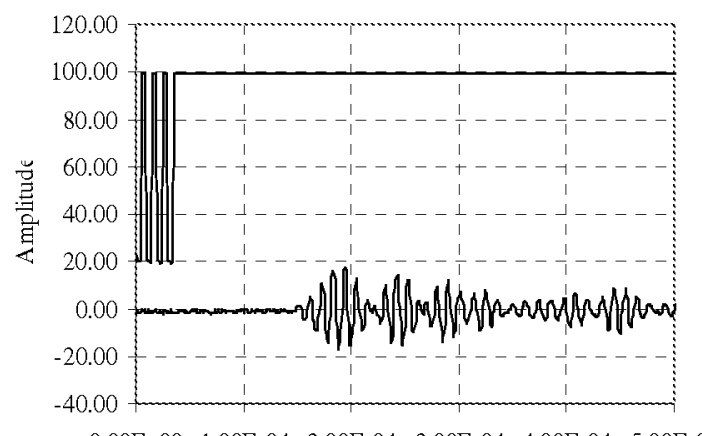

0.00E+00 1.00E-04 2.00E-04 3.00E-04 4.00E-04 5.00E-04

$t(s)$

Figure 2.2 Representation of experimental data obtained from the digital data acquisition system. Square wave corresponds to the transmitted signal and sinusoidal wave represents a received signal.

The transit time for the ultrasound pulse was determined from the correlation function

$$
K_{12}(\tau)=\overline{e_{1}(t+\tau) e_{2}(t)} \text {, }
$$

where $K_{12}(t)$ is a cross correlation function of $e_{1}$ and $e_{2}$. 
In Figure 2.3 we compare the travel-time variance with Chernov ${ }^{1}$ estimates and with theoretical estimations of second-order travel time variance by Iooss et al. ${ }^{4}$ The travel time variances are plotted in terms of the normalized distance $x / M$. The principle observation is that the departures from the Chernov prediction increase with travel distance. It shows a trend that appears to be initially linear and finally nonlinear. In addition, the curve presents the shape predicted by Iooss et al, ${ }^{4}$ who developed a theoretical model for the second-order travel-time variance that depicts nonlinear behavior at large propagation distances.

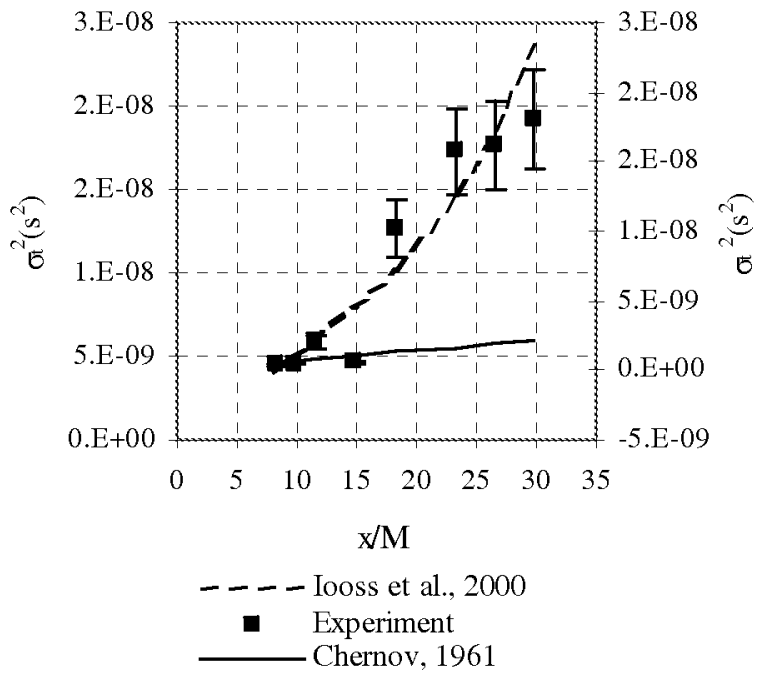

Figure 2.3 Experimental data for travel time variance plotted along with theoretical predictions by Chernov, ${ }^{1}$ and Iooss et al. ${ }^{4}$

The nonlinear behavior of the travel time variance has been observed in the literature and linked to the occurrence of caustics. The linear increase very well exhibits up to $x / M=15$ and afterwards, travel time deviations demonstrates almost quadratic growth. Exactly the same dynamics was observed in numerical experiments by Juve et al, [1991]. Probability densities for the occurrence of caustics were calculated theoretically. ${ }^{2,6,7,8,12,17}$ For our experimental data we estimate the probability density of occurrence of caustics using theory developed by Klyatskin ${ }^{12}$ and explored by Blanc-Benon et al. $^{7}$ and Iooss. ${ }^{4}$ The probability density function $P(\tau)$ for a plane wave propagating through 3D isotropic turbulence is defined as $^{6,12}$

$$
P(\tau)=\frac{\alpha}{\tau^{4}} \exp \left(-\beta / \tau^{3}\right) ; \alpha=1.74, \beta=0.66
$$

The normalized distance $\tau$ is defined as

$$
\tau=D^{1 / 3} x
$$

where $D$ is the diffusion coefficient introduced by Klyatskin. ${ }^{12}$ For a Gaussian correlation function the diffusion coefficient $D$ in a moving random medium is defined by Blanc-Benon et al. ${ }^{7}$ as

$D=\frac{\sqrt{\pi}}{2 L_{0}^{3}} \sigma_{\varepsilon}^{2} ; \sigma_{\varepsilon}=\sqrt{\left\langle\varepsilon^{2}\right\rangle} ; \varepsilon=2 n$,

where $\sigma_{\varepsilon}$ is a standard deviation of an index of refraction $\varepsilon$. It is known ${ }^{18}$ that fluctuations in the refractive index of a moving medium in the direction of the $\mathrm{x}$-axis are given by:

$$
n=-\frac{2\left(c^{\prime}+u^{\prime}\right)}{c_{0}}
$$

The standard deviation $\sigma_{\varepsilon}^{2}$ was estimated in our previous experiments ${ }^{19}$ to be at maximum 0.015 for our particular experimental conditions.

In Figure 2.4 the probability density of the occurrence of caustics is plotted along with experimental data for the travel time variance, Chernov ${ }^{1}$ theory for the linear propagation and results of the theoretical model developed by Iooss et al. $^{4}$ as a functions of normalized distance of propagation, $\tau$.

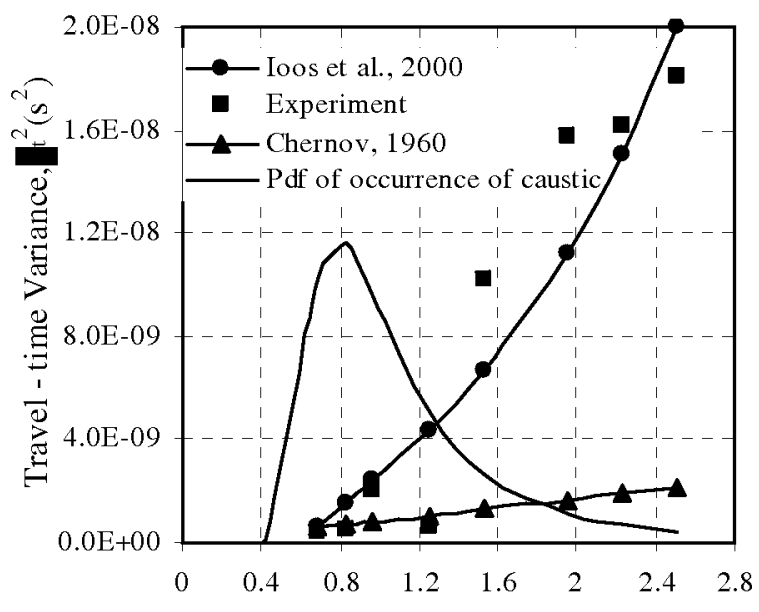

Normalized Propagation Distance, $\tau$

Figure 2.4 Probability density function of occurrence of caustic along with nonlinear dynamics of the travel time variance. 
The peak of the probability density function appears at approximately $\tau=0.82$, which corresponds to $x \approx 6 \cdot 10^{-2} \mathrm{~m}$, or in nondimensional units $x / M \cong 10$. The nonlinear behavior of the travel time variance appears right after the peak of the probability density function. Iooss et al in their theoretical modeling detected the maximum value of the probability density with their value $\sigma_{\varepsilon}=0.01$, when $x / M \cong 14.1$. We expected the maximum of the PDF to appear at a shorter distance since standard deviation in our experiment greater than one in theoretical modeling by Iooss et al. ${ }^{4}$ Given the fact that the probability density function was recovered based on just 12 discrete points the prediction is fairly accurate.

\section{CONCLUSIONS}

The importance of the presented paper is in the attempt to demonstrate experimentally the nonlinear effect of travel time variance predicted theoretically and 0numerically. Experimental data have been collected using ultrasonic technique in inhomogeneous moving medium produced by a grid placed at the beginning of a wind tunnel test section. After a linear increase (Chernov approximation), the travel time variance departs from linear behavior at a certain propagation distance due to the occurrence of the first caustics. To reinforce this point of view the probability density function associated with the occurrence of the first caustic specific for our experimental conditions has been plotted against travel-time variance versus nondimensional distance. Currently we are extending the technique to investigate the correspondence between the mean velocity in the wind tunnel and first appearance of nonlinearity in the travel time variance in order to verify the theoretical fact, that the higher the turbulent intensity, the shorter the distance at which the first caustics occurs.

\section{REFERNCES}

1 L. Chernov, "Wave propagation in a random medium," McGraw-Hill, New York, 1961.

2 Juvé, D., Blanc-Benon, Ph. And Comte-Bellot, G., "Transmission of Acoustic Waves through Mixing Layers and 2D Isotropic Turbulence," Turbulence and Coherent Structures, Métais and Lesieur, eds., Selected papers from Turbulence 89: Organized Structures and Turbulence in Fluid Mechanics, Grenoble, 18-21 September 1991.

3 Karweit, M., Blanc-Benon, Ph., Juvé, D. and Comte-Bellot, G., Simulation of the Propagation of an Acoustic Wave through a Turbulent Velocity Field: a
Study of Phase Variance," Journal of Acoustical Society of America, Vol. 89(1), 1991, pp. 52-62.

4 B. Iooss, Ph. Blanc-Benon and C. Lhuillier, "Statistical moments of travel times at second order in isotropic and anisotropic random media," Waves Random Media, 10, 2000, pp. 381-394.

5 Codona, J.L., Creamer, D.B., Flatte, S.M., Frelich, R.G. and Henyey, F.S., "Average Arrival Time of Wave Pulses Through Continuous Random Media," Physics Review Letters, Vol. 55(1), 1985, pp.9-12.

6 Kulkarny, V.A. and White, B.S., "Focusing of Waves in Turbulent Inhomogeneous Media," Journal of Physics of Fluids, Vol. 25 (10), 1982, pp. 1779-1784.

7 Blanc-Benon, Ph., Juvé, D., Ostashev, V.E. and Wandelt, "On the Appearance of Caustics for Plane Sound-Wave Propagation in Moving Random Media," Waves in Random Media, Vol. 5, 1995, pp. 183-199.

8 Blanc-Benon, Ph., D. Juvé, and G. Comte-Bellot, "Occurrence of caustics for high-frequency caustic waves propagating through turbulent field," Theoret. And Comput. Fluid Dynamics 2, 1991, pp. 271-278.

9 Iooss, B. and Galli, A., "Statistical tomography for seismic reflection data," Proceedings of the 6th International Geostatistics Congress, Cape Town, South Africa, April 2000.

10 Spiesberger, J.L., "Ocean Acousitc Tomography: Travel Time Biases, Journal of Acoustical Society of America, Vol. 77, 1985, pp. 83-100.

11 Samueldis Y. and Mukerji, T., "Velocity Shift in Heterogeneous Media with Anisotropic Spatial Correlation," International Geophysical Journal, Vol. 134, 1998, pp. 778-786.

12 Klyatskin, V.I., "Caustics in Random Media," Waves in Random Media, Vol. 3, 1993, pp. 93-100.

13 Blanc-Benon, Ph., Juvé, D. and Chevret, P., "On the influence of the turbulence modeling for atmospheric sound propagation," Journal of Acoustical Society of America, Vol. 98, 1995, p. 2924.

14 Lynnworth, L.C., Ultrasonic Measurements for Process Control, Academic Press, San Diego, CA, 1989.

15 Andreeva T.A. and Durgin, W.W., "Ultrasound Technique for Prediction of Statistical Characteristics of Grid-Generated Turbulence," AIAA Journal, Vol. 41(8), 2003, pp.1438-1443.

16 Yeh, T.T. and van Atta, C.W., "Spectral Transfer of Scalar and Velocity Fields in Heated-grid Turbulence," Journal of Fluid Mechanics, Vol. 58, 1973, pp. 233-261.

17 Kravtsov, Yu.A., "Strong Fluctuations of the Amplitude of a Light Wave and Probability of Formation of Random Caustics," Soviet Physics JETP, Vol. 28(3), 1969, pp. 413-414. 
18 Tatarski, V.I., The Effect of the Turbulent Atmosphere on Waves Propagation, Israel Program for Scientific Translation, Jerusalem, 1971.

19 Andreeva T.A., "Ultrasonic Technique in Determination of Grid-generated Turbulent Flow Characteristics", Ph.D. Dissertation, Worcester Polytechnic Institute, 2003. 